\section{LOS PROBLEMAS TEÓRICOS Y METODOLÓGICOS DEL CONCEPTO DE EXCLUSIÓN SOCIAL. Una visión neofuncionalista}

\author{
José María García Blanco \\ Universidad de Oviedo, España. \\ jblanco@uniovi.es
}

Cómo citar este artículo / Citation: García Blanco, J. M. 2016. "Los problemas teóricos y metodológicos del concepto de exclusión social. Una visión neofuncionalista", Revista Internacional de Sociología, 74 (2): e029. Doi: http://dx.doi. org/10.3989/ris.2016.74.2.029

\section{Resumen}

El éxito de la idea de exclusión social en la política social y las profesiones sociales europeas no guarda correspondencia con su claridad conceptual ni su determinación empírica. La habitual asociación de la exclusión social con la privación económica tiene mucho que ver con este problema, que además dificulta una investigación empírica acorde con los rasgos más importantes que se atribuyen a este fenómeno relacional: la pérdida multidimensional, acumulativa y secuencial de la inclusión social.

El objetivo del artículo es clarificar teóricamente la exclusión social desde un (heterodoxo) punto de vista neofuncionalista y, a partir de él, proponer una metodología de investigación que facilite una aproximación empírica más adaptada a los mencionados rasgos distintivos de este fenómeno social.
\end{abstract}

\section{Palabras Clave}

Autonomía funcional; Diferenciación social; Efectos spill-over; Funcionalismo de las equivalencias; Interdependencias funcionales.

\section{THEORETICAL AND \\ METHODOLOGICAL PROBLEMS OF THE SOCIAL EXCLUSION CONCEPT. A Neofunctionalist Approach}

Copyright: @ 2016 CSIC. Este artículo de acceso abierto distribuido bajo los términos de la licencia Creative Commons Attribution-Non Commercial (by-cn) Spain 3.0.

Recibido: 28/08/2014. Aceptado: 03/11/2015. Publicado on line: 18/05/2016.

\section{Abstract}

The success of the idea of social exclusion in European social policy and social work has no direct correspondence with its conceptual clarity and empirical determination. The usual connection of social exclusion with economic deprivation has much to do with this problem, which also hinder to make the empirical research in a form proper to the main features of this relational phenomenon: the multidimensional, cumulative and sequentially loss of social inclusion.

The aim of this paper is to make a theoretical clarification of social exclusion from a (heterodox) neofunctionalist point of view, and from it to propose a methodology of research, in order to facilitate an empirical approach more adapted to the aforementioned features of this social phenomenon.

\section{KEYWORDS}

Equivalence Functionalism; Functional Autonomy; Functional Interdependences; Social Differentiation; Spill-Over Effects. 


\section{LA GÉNESIS Y LOS PROBLEMAS DEL CONCEPTO "EXCLUSIÓN SOCIAL"}

Aludiendo con mayor o menor precisión a una serie de graves carencias sociales que demandan una respuesta urgente de la sociedad, en especial por su tendencia acumulativa en individuos, familias y territorios, el término "exclusión social" ha terminado por convertirse en el punto de referencia casi obligado de las políticas, organizaciones y profesiones sociales en Europa.

Esto se ha producido como si el término gozara de un grado de evidencia tal que no precisara de un criterio objetivable de definición, pues la mera alusión al mismo parece dar cobertura institucional y legitimidad social a políticas e intervenciones sociales que pretenden hacerle frente. Importante para ello ha sido la poderosa imagen espacial implícita en el término entre un interior y un exterior de la sociedad, que sugiere una severa fractura entre quienes no están afectados por el problema de la exclusión (y por tanto estarían "dentro" de la sociedad) y quienes sí lo están (y por tanto quedarían "fuera" de la sociedad). El resultado es que, como ha señalado Herzog (2011: 611 y ss.), la exclusión social opera más como una idea que como un concepto.

Para comprender este estado de cosas es preciso reconstruir la génesis del término exclusión social, que se remonta a los años sesenta (Kanfler 1965), aunque su empleo solo se hace frecuente con el debate suscitado por el libro de René Lenoir Les exclus (1974). En él, se presenta a los excluidos como un colectivo muy heterogéneo, formado por individuos a los que la sociedad no presta atención o viven en los márgenes de la "normalidad" social: los "inadaptados, marginales $o$ asociales [...], incapaces de atender sus necesidades, o que exigen cuidados constantes, o representan un peligro para los demás, o se encuentran segregados, bien por causa de ellos mismos, bien por causa de la colectividad" (Lenoir 1974: 10).

Los pobres como tales no son incluidos por Lenoir entre los excluidos, al considerar que la pobreza es solo un factor de vulnerabilidad entre otros, y que entre ella y la inadaptación hay más una correlación que una relación causal (1974: 27 y ss.), como probaría el hecho de que la exclusión afecta a todas las capas sociales.

Este deslinde de la pobreza obligó a Lenoir a buscar un criterio distinto de la falta de recursos como fundamento de la exclusión social, y lo encontró en una especie de patología social de raíz moral, pero que queda sin teorizar, como se pone de manifiesto en el continuo baile de términos utilizados como equivalentes para referirse a él (exclusión, inadaptación, marginalidad...) y en la gran diversidad de situaciones, cuyo denominador común es institucional: se trata de individuos que, por variadas razones, son objeto de la atención de los servicios sociales.
Los excluidos son presentados, así, como los habitantes de los suburbios marginales, los parados de larga duración, los niños y jóvenes que fracasan en el sistema educativo, los colectivos que no tienen acceso al sistema sanitario ni a los servicios y ayudas sociales, etc. Lo que vendría a poner de manifiesto en cada uno de estos casos es una línea simbólica que separa a dos grupos de población: los que viven en espacios urbanos normalizados y los que viven es espacios degradados y degradantes; los que acceden al mercado de trabajo normalizado y los que quedan fuera de él; los que se atienen a la norma educativa y los que no -y en consecuencia fracasan en la escuela-; quienes acceden a las instituciones de la seguridad social y a los servicios de salud y quienes no acceden ni a unas ni a otros. La exclusión social viene a hacer referencia, pues, a una división del espacio social: hay quienes están dentro de la sociedad -integrados en ella- y quienes están fuera -los excluidos de ella-.

El texto de Lenoir, por lo demás, no ofrece respuesta a la cuestión crucial de cómo se produce la división de la sociedad entre incluidos y excluidos, que solo bastantes años después, en la década de los noventa, es abordada de forma más rigurosa.

En 1991, un grupo de trabajo encabezado por Philippe Nasse elaboró un informe sobre la exclusión para el Comisionado del XI Plan Económico Nacional francés. En él, si bien subsiste la ambigüedad relativa a la diferencia entre exclusión e inadaptación, podemos encontrar dos ideas muy interesantes. La primera es que la exclusión simbólica es un desencadenante de situaciones de exclusión; y la segunda, que toda situación de exclusión social representa un riesgo de exclusión acumulativa, es decir, acaba generando otras formas de ruptura del lien social (Nasse 1991: 33), que es un concepto clave para entender la génesis de la noción de exclusión, que, como certeramente ha señalado Fassin (1996), remite a las tradiciones francesas de la higiene y la asistencia sociales, por un lado, y de la sociedad como un organismo cohesionado moralmente de la teoría social durkheimiana, por otro.

En un segundo informe para dicho plan, este proceso de exclusión social se focalizó en el desempleo, ya que pese a constatar "la gran heterogeneidad de las poblaciones excluidas y la diversidad de trayectorias que conducen a la exclusión", se puso especial énfasis en que "el desempleo de larga duración o recurrente se ha convertido en la manifestación y la fuente principal de exclusión" (Fragonard 1993: 16 y ss.).

Este giro hacia el desempleo como el centro de los procesos que deterioran el "vínculo" del individuo con la sociedad normalizada no impidió que la noción de exclusión social siguiera cubriendo un amplio espectro de situaciones susceptibles de ser referidas a fenómenos diversos, como la pobreza, la desigualdad o la ciudadanía. Si se reúnen todos ellos bajo un denominador común es porque se considera que, a fin de cuentas, 
desembocan en una fractura social entre quienes tienen un "lugar" en la sociedad y quienes no lo tienen.

Dentro de este proceso de difusión acelerada del término "exclusión social" durante los años noventa, tuvieron lugar los, hasta ahora, más sistemáticos intentos de teorizar la exclusión social, que tienen como marco de referencia los discursos sobre las "nuevas formas de pobreza" y la "nueva cuestión social".

En el primer caso, nos encontramos con los análisis de Serge Paugam (1991 y 1993) sobre los procesos de "descualificación social". Para Paugam, las líneas principales de fractura social que están en el origen de la nueva pobreza dependen de dos grandes desarrollos estructurales en las sociedades industriales avanzadas: la degradación del mercado de trabajo y el crecimiento, asociado a él, del desempleo de larga duración, por un lado; y, por otro, el debilitamiento de los vínculos sociales generados por la familia y la solidaridad de clase (obrera).

En el segundo caso, Robert Castel (1991 y 1995) presenta la "nueva cuestión social" en el marco de la historia de la relación entre trabajo y sociabilidad, con la que explora cómo la inserción de los individuos en la división del trabajo había permitido construir una serie de formas de cohesión social, así como las "zonas de vulnerabilidad social" que revelan el aspecto anómico de dichas formas. En relación con tales zonas, Castel no trató tanto de mostrar la localización de los individuos en ellas, como de explicar los procesos de tránsito de la integración a la vulnerabilidad o precariedad y/o de esta a la "inexistencia social".

En relación con esta última, Castel prefiere referirse más a la "desafiliación" que a la exclusión, al considerar que la primera, más que ratificar una ruptura, apunta a la reconstrucción de las trayectorias que conducen a ella; mientras que la segunda tiene una connotación excesivamente estática, por referirse sobre todo a un estado (o conjunto de estados) de privación, cuya constatación cree que dificulta, más que facilita, la recomposición del proceso que produce tal situación (Castel 1995: 13 y ss.). Es más, Castel recalca, con acierto, que la exclusión de ningún modo puede entenderse como inexistencia de relaciones sociales, sino como un conjunto de relaciones sociales particulares. Como nadie estaría fuera de la sociedad, la metáfora espacial "dentro/fuera" subyacente a la noción de exclusión sería poco adecuada para simbolizar el problema de referencia, que sería captada mejor por la diferencia "centro/periferia". Así, en la sociedad habría un conjunto de posiciones cuyas relaciones con el "centro" son más o menos laxas, siendo de él de donde surge la onda de choque que atraviesa la estructura social. Pero si el foco se dirige hacia las situaciones periféricas, tal y como hace el concepto de exclusión, cree Castel que quedan ocultos los procesos desencadenados por esa onda de presión, al igual que las continuidades de los mismos a través de la estructura social, lo que conduciría a olvidar que la vulnerabilidad golpea a la sociedad en sus relaciones laborales, a través de las diversas formas de desregulación y flexibilización del trabajo.

Los problemas de estos desarrollos teóricos relativos a la exclusión son, sobre todo, dos. En primer lugar, presentan la exclusión social como un fenómeno propio de la madura sociedad moderna, y por lo tanto dificultan la posibilidad de entenderla en toda su magnitud; es decir, como un problema presente en toda sociedad, cuyas formas y consecuencias cambian con la transformación de las sociedades. $Y$ en segundo lugar, el uso de una concepción estratificatoria de la estructura social moderna, aunque se presente en forma de una diferencia centro/periferia social, conduce a una combinación poco clara de conceptos marxistas (explotación laboral) y weberianos (dominación y cierre social), que se traduce en la idea de que el problema de la desafiliación social radica en si las estructuras ocupacionales y las instituciones políticas que distribuyen recursos y oportunidades sociales permiten a la sociedad ofrecer "sitio" a todos o, por el contrario, hacen que sufra un déficit estructural de "sitios", de "lugares sociales" (Laparra et al. 2007: 18 y ss.).

Este planteamiento teórico no ha sido óbice, sin embargo, para que sus partidarios hayan señalado que tanto el "espacio" de la exclusión como las "trayectorias" de desafiliación/descualificación social que conducen a él originan un fenómeno multidimensional y heterogéneo, además de esencialmente dinámico (Laparra y Pérez 2008), lo que es correcto pero contradictorio con la imagen unidimensional (focalizada en la economía, particularmente en el ámbito laboral-ocupacional) y compacta (ejercicio de la dominación y clausura social) que proyecta dicho planteamiento. Podemos preguntarnos, por ejemplo, cómo es posible explicar desde esta perspectiva que una persona de clase media con una carrera profesional normalizada, a causa de una adicción dañe gravemente sus relaciones familiares, deteriore severamente su estatus laboral y acabe refugiándose en círculos formados por personas en situación similar, lo que a su vez con frecuencia realimenta su proceso de exclusión familiar y laboral.

Este ejemplo creo que clarifica, no solo que los fenómenos de exclusión no dependen de las categorías marxistas enraizadas en el concepto de explotación, como señaló hace ya tiempo Rosanvallon (1995: 7), sino, también, que es difícil entenderlos a partir de las categorías de dominación y cierre social, pilares fundamentales de la teoría weberiana de la estratificación (Silver 2007). Por ello, se hace necesaria una teorización alternativa de la exclusión social, capaz de articularla dentro de una teoría general de la sociedad y su evolución, así como de dar cuenta coherente del carácter "dinámico", "multidimensional" y "heterogéneo" que hoy se le atribuye de forma generalizada. 


\section{FUNDAMENTOS NEOFUNCIONALISTAS DE UNA ALTERNATIVA TEÓRICA}

El título de neofuncionalista con el que hago referencia a mi propuesta de análisis alternativo de la exclusión social, alude a una corriente del pensamiento sociológico que en los años setenta del pasado siglo retomó la inspiración funcionalista, tras el fuerte descrédito en que había caído su versión clásica durante la década anterior. Esta denominación, como ha señalado Jeffrey C. Alexander (1985), se acuñó a imagen y semejanza del rótulo neomarxismo, utilizado para referirse al amplio movimiento intelectual que, poco antes, había llevado a cabo una renovación sustantiva del pensamiento marxista clásico.

Como el neomarxismo, el neofuncionalismo realizó una crítica decidida de algunos supuestos básicos del planteamiento original, incorporó algunos elementos de otras corrientes intelectuales supuestamente antagónicas y no se materializó en una perspectiva única, sino en una diversidad de desarrollos teóricos frecuentemente conflictivos entre sí. De ellos, será el construido por el sociólogo alemán Niklas Luhmann el que sirva de referencia a mi propuesta teórica.

Como es bien sabido, el funcionalismo clásico antepuso el concepto de estructura al de función, pues presuponía unos sistemas sociales provistos de determinadas estructuras $\mathrm{y}$, a partir de ello, se interrogaba por sus prestaciones funcionales, que debían ser atendidas si los sistemas en cuestión debían conservarse (Radcliffe-Brown 1972; Parsons 1964). A este enfoque estructural-funcional, Luhmann contrapuso una alternativa a la que denominó funcional-estructuralismo, porque su idea central es la necesidad de invertir los términos de la relación entre estructura y función (Luhmann 1974a). Luhmann consideraba que, más allá de la tradicional crítica por la dificultad de explicar el cambio y el conflicto sociales, el principal problema del estrucural-funcionalismo residía en esa anteposición por principio de las estructuras a las funciones, pues creía que cerraba la posibilidad de problematizar las estructuras y plantearse su sentido para la constitución y reproducción de los sistemas sociales.

Con este giro en la fundamentación del análisis funcional, su punto de referencia ya no es un estado de cosas dado (un sistema estructurado y su conservación), sino algo más abstracto y abierto: el mundo y su complejidad, entendida como totalidad de lo posible en él. Y como el concepto de posibilidad presupone que es factible condicionar y limitar lo posible, los sistemas son concebidos como aquellas unidades que, dentro del mundo, a través de sus estructuras convierten lo posible en algo determinado, o cuando menos determinable.

Sistemas sociales y personales procesan la complejidad del mundo de una forma peculiar: erigiendo condicionantes y limitaciones que seleccionan deter- minadas posibilidades, pero a la vez permiten referirse a otras y ordenar el acceso a las mismas. En el plano social del mundo, la complejidad se presenta como doble contingencia, de la que se deriva una tendencia a la indeterminación reflexiva de la interacción social. A tal efecto, los sistemas sociales disponen de dos vías diferentes pero complementarias de estructuración: la generalización de expectativas y la diferenciación interna, pues mediante ellas la sociedad reduce y conserva complejidad de una forma ordenada, al facilitar la selección de acciones concretas entre las socialmente posibles, pero remitiendo a la vez siempre a nuevas posibilidades de acción, que exigirán una nueva selección.

Estos dispositivos de estructuración social no pueden ser entendidos como lo fijo o estable de la sociedad, por contraposición a unos procesos sociales que representasen su fluidez y variabilidad. La incesante sucesión de fenómenos sociales, que a cada instante convierte un futuro que siempre mantiene abiertas distintas posibilidades en un pasado ya inmodificable, implica por sí misma una reducción fáctica de complejidad. Justo de ello y de la consiguiente necesidad de delimitación sistémica es de lo que da cuenta el concepto de proceso social. En pocas palabras: los procesos son sistemas y precisan una estructura, cuya función no consiste en eliminar la selectividad espontánea de los procesos, sino en reforzar su capacidad selectiva, facilitando así su delimitación y una mejor determinación de su funcionamiento.

De esta diferencia fundamental resulta otra de especial relevancia para el tema de este artículo: la relativa al concepto de "evolución social", con el que se debe dar cuenta de cómo emergen y se reproducen las estructuras de la sociedad.

La explicación de cómo emergen y se reproducen las estructuras sociales adopta en Parsons (1974: 40 y ss.) más la forma de un proceso de desarrollo que propiamente evolutivo, al entender la evolución social como un proceso de progresiva diferenciación de las estructuras sociales que deben atender cada una de las cuatro funciones analíticamente identificadas por su teoría general de la acción con el famoso esquema "AGIL".

Frente a este esquema "desarrollista" de la evolución social, Luhmann toma como punto de partida la idea de que el análisis evolutivo debe explicar cómo el cambio social hace posible que de la entropía social pueda surgir la neguentropía; es decir, cómo tiene lugar la morfogénesis de la complejidad social (Luhmann 1997: 414).

Luhmann busca la respuesta a esta cuestión en el neoevolucionismo, en el que la morfogénesis de la complejidad no se explica mediante una "ley" ni apelando a las ganancias de "racionalidad" que genera la complejidad, sino suponiendo que la evolución es recursiva, ya que se concibe como un 
proceso que aplica a sus resultados el mismo procedimiento que los ha producido: la "cooperación no coordinada" de las funciones evolutivas de la variación, la selección y la reestructuración. Esta falta de coordinación sistémica (comúnmente descrita como "azar") implica que no es posible prever si las variaciones conducirán a una selección positiva o negativa de las novedades que presentan, y menos aún si se producirá la reestructuración a partir de la correspondiente selección. El resultado de ello es una concepción de la evolución como un proceso interminable que tiene lugar en un tiempo irreversible, en cuyo curso cualquier reestructuración estabilizadora se convierte en punto de apoyo para la génesis de nuevas variaciones $-\mathrm{y}$ esto, lógicamente, tanto más cuanto mayor sea la complejidad alcanzada-.

Al representar tanto el final de una secuencia evolutiva como el presupuesto de la nueva variación social, la reestructuración evolutiva simboliza la unidad de la morfogénesis social y ha de ser concebida como "estabilización dinámica". Su soporte fundamental son las ya mencionadas formas de diferenciación interna de la sociedad, a saber: la segmentaria, característica de las sociedades arcaicas; la estratificatoria, propia de las sociedades de castas y estamentales; y la funcional, correspondiente a las sociedades modernas.

Por tanto, la evolución social no cabe ser interpretada ni como progreso ni como desarrollo, pues en ella caben por igual la emergencia de los órdenes sociales y su destrucción. La teoría de la evolución tampoco puede proporcionar un modelo interpretativo del futuro, ya que no presupone una teleología histórica (sea positiva, sea negativa) ni encierra un modelo de regulación que pudiera auxiliarnos a responder a la cuestión de si debe dejársela operar libremente o debe ser corregida. Ella solo responde a una única cuestión: cómo es posible que puedan emerger sistemas cada vez más complejos y, eventualmente, por qué fracasan.

\section{INCLUSIÓN Y EXCLUSIÓN DESDE UN PUNTO DE VISTA FUNCIONAL-ESTRUCTURAL}

La sociedad necesita de la vida consciente para existir, pero a la vez ella produce las condiciones que permiten la reproducción de esta. $Y$ es a esta conexión mutuamente constitutiva, vista desde el lado de la sociedad, a lo que nos referimos cuando hablamos de inclusión. La inclusión alude, pues, a que la sociedad ha de aprovechar la complejidad de la vida consciente para producir interacción social. Sin embargo, la complejidad de esta conexión también produce exclusión, pues para que la inclusión sea posible los seres humanos deben ser distinguidos por sus respectivas aportaciones a la interacción social. Es decir, participar en la interacción social requiere que los individuos haga- mos nuestras propias contribuciones -corporales y psíquicas-, y así debamos distinguirnos unos de otros, comportándonos de forma mutuamente exclusiva, pues solo así es posible realizar nuestra propia aportación a la reproducción de la sociedad. Justo porque hemos de cooperar, en contra de cualquier analogía natural, ha de estar claro quién realiza cada contribución.

Este es el fondo a partir del cual, en conexión con la teoría de la división social del trabajo y la distinción entre solidaridad mecánica y orgánica, Durkheim sostuvo que las sociedades modernas, al estar provistas de un mayor grado de división del trabajo y una forma orgánica de solidaridad, producen a la vez más inclusión y más exclusión (recíproca), dando origen a su conocida tesis de que las sociedades industriales impulsan una creciente "individualización" de las personas, con su secuela de una creciente anomia.

Siguiendo la estela de Durkheim, e incorporando la teoría del desarrollo de la ciudadanía de Thomas H. Marshall, Talcott Parsons (1973: 306) definió la "inclusión" como un conjunto de patrones de acción que individuos y/o grupos deben seguir para ser aceptados como miembros efectivos de un sistema de solidaridad social. Lo que a Parsons le interesaba era, sobre todo, el proceso de inclusión en las unidades sociales más grandes y complejas que produce el proceso evolutivo de las sociedades, y que representaría un requisito para la creciente diferenciación que caracteriza a las más evolucionadas (1971: 92 y ss.). Con el progreso de la diferenciación social, las condiciones de la inclusión cambiarían; de manera que en las más diferenciadas y complejas sociedades modernas dichas condiciones ya no pueden ser tan compactas ni ordenarse tan jerárquicamente como en las sociedades premodernas, por lo que han de hacerse mucho más individualizadas. Las sociedades modernas se caracterizarían, así, por ofrecer posibilidades de inclusión a todos los individuos, y el problema se reduciría a cómo condicionar estas posibilidades y hacerlas efectivas -es decir, a cómo articular igualdad y desigualdad según el éxito y el reconocimiento social (1977)-.

Resulta evidente que esta concepción de la inclusión es excesivamente simplista, al faltarle una reflexión acerca del caso negativo de estas categorías, que solo puede subsanarse introduciendo la distinción entre inclusión y exclusión. Si la inclusión representa la chance de un individuo de ser reconocido o relevante a efectos de interacción social, o dicho de un modo más convencional, de ser tratado como "persona" (Luhmann 1995: 241), entonces no es más que la cara interna de una diferencia, pues si existe inclusión es porque la exclusión -el no reconocimiento como "dirección" interactiva, como "persona"- también es posible. Más aún, la cohe- 
sión social puede ser observada y sus condiciones especificadas solo gracias a la existencia de individuos y grupos que no participan de ella (Geremek 1976: 11). Es decir, si las condiciones de la cohesión y la inclusión sociales pueden ser especificadas como la forma del orden social es porque la exclusión representa la contraestructura del sentido y fundamento de dicho orden.

En contra de lo sostenido dentro del funcional-estructuralismo por Stichweh (2005) y fuera de él por algunos de sus críticos (Herzog 2011: 615 y ss.), la diferencia inclusión/exclusión no puede entenderse como una oposición jerárquica, en el sentido de Dumont (1970); es decir, como una diferencia en la que el valor negativo no es más que una forma subordinada al valor positivo, que por eso engloba a ambos. Esta proposición es adecuada para entender la lógica de la diferenciación estratificatoria, en la que el valor negativo (plebeyo, impuro) designa una forma de inclusión subordinada al término positivo (noble, puro), el cual puede entonces actuar a la vez como parte y como representante del todo (de la diferencia misma). El problema, como veremos de inmediato, es que la exclusión en las sociedades estratificadas hace referencia, justo, a aquellos que quedan fuera del régimen de clasificación jerárquico, por caracer de familia u hogar o por ser "intocables"1. Pero es que, además, cuando la heterárquica sociedad moderna instituye -dentro de su sistema político- planes, programas y servicios relativos a la diferencia inclusión/exclusión, el término operativo es justamente el valor negativo, pues solo a partir de la exclusión puede producir y conectar sus operaciones inclusivas, mientras que el positivo queda convertido en un valor que solo orienta la reflexión acerca de la contingencia de los resultados de dichas operaciones.

A esta forma de entender inclusión y exclusión se le ha reprochado, en el contexto de su aplicación a las sociedades modernas, que "su radicalidad teórica la hace inviable", porque "prácticamente no se encuentran casos de una exclusión (total) de sistemas" (Herzog 2011: 617). Sin embargo, esta crítica está fundamentada en una lectura poco atenta del planteamiento de Luhmann, pues ignora que, para este, si bien la distinción inclusión/exclusión tiene un sentido propio, y como tal es definida y analizada en el marco de su teoría general de la sociedad, su materialización histórica está estrechamente relacionada con la forma dominante de diferenciación de la sociedad. Por eso, en la sociedad moderna, al ser inclusión y exclusión producidas por los diferentes subsistemas funcionales, y no por el sistema global de la sociedad, "la diferencia inclusión/exclusión nunca se da empíricamente con tanta claridad como para que todas las personas puedan ser inequívocamente clasificadas en uno de sus lados" (Luhmann 1995: p. 263).

\section{INCLUSIÓN, EXCLUSIÓN Y DIFERENCIACIÓN SOCIAL}

Para el funcional-estructuralismo, a diferencia de los enfoques más habituales de la exclusión social, esta no es un fenómeno subordinado ni privativo de las sociedades postindustriales -en tanto que nueva forma de pobreza o nueva cuestión social-. En cuanto reverso de la inclusión, la exclusión está presente en todo orden social, y por ello varía con la evolución sociocultural, dependiendo, sobre todo, de las distintas formas de diferenciación social que van apareciendo en su curso.

En las sociedades arcaicas, diferenciadas conforme a líneas de segmentación según el parentesco, la inclusión resulta directamente de la pertenencia a un grupo de parentesco (Evans-Pritchard 1997: cap. 3). Esta forma de inclusión hace muy difícil la exclusión, pues la pérdida de la pertenencia a un segmento puede ser atenuada por la inclusión en otro, como ocurre en el caso de la mudanza y traslado a otra tribu. La exclusión pura y dura era un fenómeno marginal, por lo común asociado al destierro, que castigaba la infracción de una norma fundamental de convivencia y se materializaba generalmente de una forma impetuosa, debido a la falta de un sistema jurídico diferenciado (Lienhardt 1958; Posner 1980).

En las más evolucionadas sociedades estratificadas, la inclusión está determinada por el correspondiente sistema de estratos y rangos sociales. Cada ser humano tiene aquí una posición social diferenciada y su correspondiente dignidad personal en función del estrato al que pertenece. Pero la regulación de inclusión y exclusión se continúa produciendo en el plano segmentario, al componerse los estratos sociales de unidades familiares. Cada individuo obtiene su estatus social en virtud de la familia en la que ha nacido o, en el caso de los servidores, por el hogar en el que prestan sus servicios. Excluidos, por tanto, son aquellos que carecen de una familia o de un hogar reconocidos; en el caso de la sociedad medieval y de la temprana modernidad europea, por ejemplo, los mendigos, el personal de conventos y monasterios (según la posición social), quienes ejercen profesiones deshonestas, así como gran parte de la tropa de los ejércitos y del personal de la marina (Geremek 1976 y 1998; Piergiovanni 1993).

Este espacio de exclusión, que se amplía según avanza el proceso de decadencia del orden agrario medieval, es especialmente reconocible porque en relación con él quiebran las normas tradicionales de reciprocidad, de forma que algún tipo de solidaridad solo podía mantenerse en virtud de ideas religiosas acerca del deber de compasión y la salvación de la propia alma, lo que movía a los excluidos al empleo de todo tipo de trucos y engaños para obtener compasión.

La diferencia inclusión/exclusión se reconstruye ahora dependiendo de la sedentariedad, que permi- 
te mantener relaciones sociales regulares $y$, a partir de ellas, formarse expectativas fiables acerca de los otros. Pero esto precisamente requiere de la existencia de exclusiones, que la sociedad no puede ignorar, aunque solo sea manteniendo ciertos tipos de conexiones secundarias. Es así como en el ámbito marginal se produce el reclutamiento de tropas para los ejércitos y de personal para servir en la marina, y los artesanos itinerantes, aunque no eran excluidos en sentido estricto, desempeñaban un importante papel en ciertos mercados de trabajo. En la temprana modernidad, las ciudades y los emergentes Estados reaccionaron a esta situación con la organización del trabajo y la organización eclesiástica, principal responsable del sistema de auxilio social (Pound 1988).

Con el cambio estructural que la modernidad representa hacia una forma de diferenciación funcional, inclusión y exclusión experimentan una transformación radical. Dado que la diferenciación funcional no distribuye a los individuos como tales entre los subsistemas, la inclusión no puede producirse ya en virtud de la pertenencia exclusiva a "un" subsistema que otorgue "una" posición social, sino que todos los individuos tienen que poder participar en todos los subsistemas sociales, y con ello ocupar múltiples y diversas posiciones para incluirse ("inclusión universalista"). Además, la inclusión tiene que hacerse según la función y los códigos comunicativos de los que en cada caso se trate, por lo que los individuos han de cambiar continuamente su registro de conducta, adaptándose a las exigencias diversas de cada contexto funcional.

Con este cambio estructural, la sociedad deja de ofrecer a los individuos un estatus social que los identifique personalmente, según su origen y condición. Si en la sociedad estratificada la orientación primordial del individuo era hacia otros roles propios -y a la dignidad personal resultante de la totalidad de los mismos-, en la moderna debe hacerlo primordialmente hacia los roles complementarios que otros desempeñan en un determinado ámbito funcional. Por eso, la inclusión depende ahora de oportunidades sociales muy diferenciadas, cuyas conexiones ya no son seguras ni susceptibles de coordinarse establemente. Se supone que todos los individuos deben poder acceder a todos los subsistemas (producir y consumir, tener y ejercer derechos subjetivos, ser ciudadanos, acceder a la educación, disponer de atención médica, formar libremente su propia familia, etc). Y cuando alguien no hace efectiva su inclusión, la sociedad tiende a imputarle el no conseguirlo, así como las consecuencias resultantes. De esta manera la sociedad se evita el considerar que la exclusión es un fenómeno que obedece a factores radicados en su estructura.

Si la inclusión se concibe de este modo, sin exclusión, en cuanto pertenencia del ser humano como un todo en la sociedad, el resultado es una lógica totalizadora que viene a ocupar el lugar de las viejas lógicas clasificatorias según la segmentación o la estratificación. La nueva lógica social exige la eliminación de su contrario, o sea, requiere unidad: todos los individuos han de ser hechos seres humanos plenos, y como tales ser provistos de derechos y oportunidades efectivas de ejercerlos.

Pero como no puede ignorarse la evidente desigualdad de condiciones de vida, esta lógica totalizadora acaba conduciendo a otra temporal. Así, se confía en el crecimiento económico, con la esperanza de que una mayor abundancia permita en el futuro una mejor distribución de las oportunidades $-\mathrm{y}$ si se considera vana tal esperanza, se apela a las revoluciones-. O se aumentan las partidas presupuestarias dedicadas a los servicios sociales y a la cooperación al desarrollo, para que los retrasados de aquí y de allá puedan recuperar el terreno perdido. En el marco de esta lógica totalizadora, las exclusiones han sido contempladas, pues, como problemas de "restos" que no la cuestionan.

Además, si en las sociedades estamentales la inclusión se determinaba mediante la posición social ocupada en el orden de los rangos, de forma que nadie debía enfrentarse a situaciones en las que debiera dar cuenta de quién era -entre las élites bastaba con decir el nombre; en los estratos inferiores, con ser conocido en los lugares en donde se vivía-, en la densamente urbanizada e impersonal sociedad moderna lo típico es justo lo contrario: hay que contar con situaciones en las que es preciso aclarar quién se es; situaciones en las que hemos de enviar señales de sondeo y observar si los otros están en condiciones de apreciar correctamente con quién se están relacionando. Por eso la identidad se convierte en un problema de autodefinición y autorrealización. Y por eso mismo se ha de distinguir entre existencia psicofísica e identidad social, lo que significa que nadie puede realmente saber con certeza quién es, teniendo que descubrir si sus propias proyecciones de identidad son reconocidas por los otros.

A partir del siglo XVIII, esta nueva situación de la inclusión es codificada con la semántica de los derechos humanos. Esta tiene dos puntos de referencia fundamentales: el negativo, en las viejas diferencias y distinciones estamentales, y el positivo, en la pretensión de sintetizar las condiciones de inclusión en el conjunto de los diversos sistemas funcionales de la sociedad mediante un principio "humano" que es neutral ante las diferencias. De esta forma, la nueva semántica inclusiva idealiza como postulados valorativos la libertad y la igualdad.

La función manifiesta de estos derechos es reconocer y ratificar ciertos hechos "naturales". Pero su función social más importante es latente: abrir por principio el futuro contra cualquier predeterminación distributiva o clasificatoria de los seres humanos, según criterios de una mayor o menor dignidad/huma- 
nidad. Proclamar la libertad e igualdad de todos los seres humanos significa, pues, extender una especie de cheque en blanco para el futuro (Luhmann 1997: 1076), dado que como tales es evidente que no han conseguido realizarse.

También en el campo semántico de los derechos humanos la exclusión se mueve por una vía secundaria y oscura, que transcurre en paralelo a la luminosa vía principal de la inclusión. El problema es que la semántica de los derechos humanos solo parece poder observar que todavía no se han realizado ni, sobre todo, se han difundido suficientemente. Al mismo tiempo, desacopla las causas de la exclusión de las semánticas normativas. Delitos, faltas y desviaciones de las normas en general no conducen ahora a una exclusión de la sociedad, que entonces ha de cargar con el problema correspondiente, y disponerse a tratarlo mediante técnicas terapéuticas y dispositivos de control de las consecuencias; lo que, con buen criterio, e inspirándose en Michael Foucault, ha denominado Cornelia Bohn (2001) "exclusión incluyente"2.

El problema de la exclusión queda así oculto por el postulado de una inclusión plena de todos los seres humanos en la sociedad. Bajo el supuesto del acceso igual y libre a los sistemas funcionales, la participación de los individuos puede producir la ilusión de que se ha alcanzado un nivel desconocido de inclusión. Pero el problema no se reduce a la inevitable discrepancia entre lo normativamente postulado y lo conseguido o a una cuestión de más o menos participación ${ }^{3}$, dado que radica en el hecho de que la forma funcional de diferenciación está marcada por una "diabólica" indeterminación congenita en lo relativo a la inclusión/exclusión (Fuchs 2000: 161), de modo que, si bien la inclusión universal es una exigencia inexcusable para ella, lleva a la vez inscrita en sí misma la inevitable producción de daños excluyentes, aunque no cumplan cometido funcional alguno.

La diferenciación estructural de la sociedad en sistemas funcionales implica que los seres humanos ya no pueden situarse socialmente a partir de su adscripción a "un" subsistema social, lo que invalida el uso de metáforas espaciales (lugar, sitio social) para entender cómo funcionan inclusión y exclusión en nuestra sociedad. Como individuos existimos fuera de dichos subsistemas, aunque como tales hemos de tener acceso a todos ellos para vivir de forma normalizada, es decir, para estar incluidos. Por su parte, estos subsistemas están en principio abiertos a todos los individuos, pero solo a través de aquellos aspectos que son relevantes para la función social en torno a la que gira en ellos la interacción social.

Esta autonomía funcional de los subsistemas, sin embargo, no significa que sean indiferentes o estancos los unos con respecto a los otros. En todos los sistemas funcionales operan mecanismos (confianza, reputación, crédito, formación, motivación, vocación, etc.) que tratan, en los términos y condiciones que determina la correspondiente lógica funcional, las consecuencias de si, y cómo, se participa en otros ámbitos funcionales, lo que redunda en una amplificación -positiva o negativa- de las desviaciones registradas más allá de su dimensión originaria, generando trayectorias y situaciones de exclusión diversas, dependiendo de dónde se originan y de cómo y hasta dónde llega la amplificación.

Estos "efectos spill-over" (Fuchs y Schneider 1995) determinan que la exclusión de hecho de un sistema funcional limite con frecuencia lo que se puede conseguir efectivamente en otros, y constriñe a una parte de la población, que puede acabar diferenciándose en espacios propios (de exclusión, marginales). Estos espacios diferenciados contribuyen al desarrollo de formas de vida e interacción social en las que los presupuestos estructurales y culturales que organizan las conductas sociales "normales" ven menoscabada su decisiva relevancia, quedando liberadas de cualquier horizonte temporal de referencia en beneficio de la inmediatez de la situación presente. De este modo, las habitualmente dilatadas expectativas de reciprocidad que ayudan a articular las relaciones sociales en el ámbito de la inclusión decaen, al punto de descomponer muchas veces los mismos vínculos sociales primarios.

Sin embargo, al llevar inscrita la inclusión universal en su forma de diferenciación, la sociedad moderna ha tenido que desarrollar instituciones destinadas a reparar y prevenir los daños excluyentes que esta misma forma de diferenciación funcional produce constantemente: las políticas y servicios sociales. El problema es que la propia diferenciación funcional restringe severamente el alcance de cualquier mecanismo de coordinación positiva entre los sistemas funcionales (Stichweh 2005: 196); es decir, de instituciones que intenten regular o controlar la transformación de las ventajas o desventajas resultantes de lo que sucede en un sistema funcional en facilidades o en obstáculos para la participación en otros. Pese a la continua exigencia realizada en este sentido al sistema político, este es solo un sistema funcional entre otros, no un complejo institucional superior o central del orden social moderno. A pesar de lo que prometen las ideas de ciudadanía social y derechos sociales, políticas y servicios sociales chocan con la decisiva limitación de no poder acceder directamente a los mecanismos de inclusión/exclusión de los sistemas funcionales ni poder forzar eficazmente su funcionamiento más inclusivo o sustituirlos, por lo que no pueden hacer efectiva, en representación de la sociedad, no ya la inclusión universal, sino tan siquiera su oportunidad. Como ha señalado Peter Fuchs (2000), políticas y servicios sociales solo pueden aspirar a generar/regenerar las condiciones contextuales que hagan posible dicha oportunidad. 
La exclusión social moderna, en definitiva, está decisivamente condicionada por la diferenciación funcional de la sociedad, de forma que esta, como sistema total, no puede regular la inclusión ni excluir a las personas.

A este planteamiento se le reprocha que, al pensar la exclusión en términos funcionales, pierde de vista que existe la exclusión total de las personas físicas de una sociedad, considerando que tal es el caso de los inmigrantes no legales que son deportados o repatriados (Herzog 2011: 617). A este respecto, debe señalarse que, como apunta pero rechaza Herzog, el funcional-estructuralismo entiende la sociedad moderna como una sociedad constitutivamente mundial, desestimando el convencional concepto de sociedad como unidad estatalmente organizada, y por ello materializada en múltiples ejemplares, según las líneas de segmentación (estatal) del sistema político mundial. En este sentido, toda deportación, repatriación o expulsión no significa exclusión "de la sociedad' como sistema", sino la imposición de un "traslado a otro segmento del sistema político de la sociedad mundial" -en el que, desde luego, suelen darse peores condiciones de inclusión-, de forma que instituciones y autoridades políticas puedan desentenderse mejor del problema, al hacer a los deportados, repatriados o expulsados más invisibles aún que la población local sometida a una situación de guetización o marginalización residencial.

\section{Consecuencias metodológicas: el FUNCIONALISMO DE LAS EQUIVALENCIAS}

Como cualquier otro fenómeno social, la exclusión es susceptible de explorarse empíricamente mediante técnicas cuantitativas y cualitativas. En el caso de las primeras, el empleo de encuestas generales, por su propia naturaleza, tiene dificultades para acceder a los colectivos sociales más excluidos, dadas sus particulares condiciones y estilos de vida, de manera que la información así obtenida permite dibujar un retrato del espacio de la vulnerabilidad más que de la exclusión social en sentido estricto (Subirats 2004: 87 y ss.). Algunas investigaciones intentan superar esta limitación mediante un diseño muestral específicamente dirigido a conseguir un mejor acceso a los sectores más desfavorecidos de la población, asignándoles una sobrerrepresentación muestral (Laparra y Pérez 2008: 213 y ss.).

Atenuado así este problema, el uso de encuestas generales puede proporcionar información relevante sobre la incidencia, distribución e intensidad de la exclusión en una población y momento dados. Pero no resuelve el otro gran problema de esta aproximación empírica: cómo abordar el decisivo aspecto dinámico de la exclusión social; es decir, el conocimiento de los procesos que la van configurando como un específico fenómeno social. Ciertamente, este problema puede ser paliado mediante una investigación de panel, pero su elevado coste y la gran dificultad de mantener una muestra lo suficientemente homogénea en las distintas oleadas, dadas las mencionadas características de la población excluida, suelen disuadir de usar este diseño destinado a producir información longitudinal (ibíd.: 189 y ss.).

La alternativa más asequible y rigurosa para resolver este segundo problema es el empleo de técnicas cualitativas como las historias de vida personal y/o familiar, muy utilizadas, sobre todo, para acercarse a colectivos excluidos concretos, pero en muchos casos desconectada de -cuando no directamente hostil a- las investigaciones cuantitativas, perdiendo así la posibilidad de obtener de ellas un rendimiento mayor: el que producirían si, sobre la base de los resultados obtenidos mediante técnicas cuantitativas, se seleccionaran individuos y hogares representativos de situaciones características dentro del heterogéneo espacio de la exclusión social, para utilizar a continuación con ellos técnicas biográficas, a fin de obtener información en profundidad sobre las diferentes trayectorias vitales que están detrás de las situaciones de exclusión social.

Así pues, la combinación ordenada y teóricamente fundamentada de técnicas cuantitativas y cualitativas, no solo es recomendable sino necesaria para atender al decisivo carácter procesal, dinámico, de la exclusión social. Y a tal efecto, entre los diversos estudios que han ensayado esta vía de investigación, tomo como punto de referencia el diseño empleado hace ya tiempo en un estudio de la pobreza en la región italiana del Veneto (Mauri y Micheli 1992), dado que la definición que se hace en él del concepto de pobreza es muy próxima a la que se ha realizado aquí del concepto de exclusión, y que sus reflexiones metodológicas nos llevan directamente al núcleo de la concepción metodológica del funcional-estructuralismo.

Los investigadores italianos estructuraron su estudio en dos líneas básicas y tres niveles de investigación. La primera línea era una encuesta general realizada mediante un cuestionario estructurado a una muestra bietápica de familias. El objetivo era reconstruir las estrategias utilizadas para afrontar los problemas que suelen surgir a lo largo del ciclo vital de una familia normal. La encuesta permitió identificar y comparar las familias "problemáticas" y las "no problemáticas".

La segunda línea de investigación estaba dirigida a una muestra no probabilística de familias "problemáticas" y usuarias de algún servicio o beneficiarias de alguna prestación social. Estas familias se clasificaron en cuatro grandes conglomerados, asociados con otros tantos factores de empobrecimiento que habían sido identificados en la encuesta general. Dentro de estos grandes conglomerados se realizaron entrevistas con un cuestionario estructurado, pero también se llevaron a cabo entrevistas no estructuradas, en las que se abordaron los concretos problemas económi- 
cos de las familias, los de organización familiar y las situaciones de crisis por las que habían atravesado las familias. Las personas de referencia pudieron dar cuenta así de cómo se habían producido dichas situaciones en sus familias, cómo habían respondido a ellas y cuáles eran sus expectativas de futuro.

La organización del estudio en estas dos líneas y tres niveles de investigación permitió a los investigadores italianos comparar diferentes segmentos de población en relación con las formas de experimentar y afrontar tensiones estructurales y existenciales. A partir de ello, la atención se focalizó en los aspectos dinámicos del empobrecimiento y en un detallado análisis de los factores diferenciales, para sacar a la luz una hipotética deriva hacia una serie de problemas característicos y las nuevas formas de superviviencia originadas por el empobrecimiento.

La investigación puso de manifiesto diferentes aspectos que, a primera vista, parecen perfectamente extrapolables al fenómeno de la exclusión social, como que el amalgamamiento de los factores de empobrecimiento con otros sucesos, condiciones y conductas puede crear combinaciones que con frecuencia son difíciles de desentrañar causalmente; o que el factor temporal puede ser decisivo en el tránsito de la normalidad a la pobreza, en la medida en que los acontecimientos se encadenan en un proceso que reduce de forma progresiva los grados de libertad de las familias, debido al agotamiento de sus recursos materiales y sociales.

Pero más allá de esta bien articulada combinación de escenarios cuantitativos generales con biografías cualitativas particulares, para poder construir un dispositivo de investigación acorde con el carácter dinámico de la pobreza, los investigadores italianos llevaron a cabo una interesante y oportuna reflexión metodológica acerca de la causalidad en la investigación social, que es también relevante para el caso de la exclusión social.

Como ha señalado una de las investigadoras (Zajczyk 1995), cuando se considera que investigar en clave de proceso un fenómeno social es decisivo para explicarlo, por entender que su carácter es esencialmente dinámico, resulta casi automático pensar en una cadena de relaciones causa-efecto. Sin embargo, Zajczyk considera que no es posible hablar de una etiología de la pobreza en sentido estricto, porque el empobrecimiento no puede ser descrito como el resultado de una sola y bien determinada cadena causal. $Y$ esto se debe a dos razones relacionadas entre sí. Por un lado, diferentes formas de pobreza pueden ser el resultado de condiciones de partida similares (principalmente, en el caso de familias que viven cerca del umbral de la pobreza). Por otro lado, una situación final similar (como la pobreza severa) puede ser producto de diferentes cadenas causales, cada una de las cuales es condición suficiente pero en ningún caso necesaria de dicho destino.
Esta es la razón que lleva a Zajczyk a afirmar que el objetivo de la investigación de la pobreza no puede consistir en determinar las causas de la pobreza. En vez de ello, y apoyándose en concepción neo-humeana de la causalidad propuesta por J. L. Mackie (1965), Zajczyk propone un sentido alternativo de dicha investigación: la búsqueda de inus-causas; es decir, la identificación de partes necesarias pero en sí mismas insuficientes de una condición que no es necesaria pero sí suficiente en un particular proceso de empobrecimiento. Puesto en términos de exclusión, esto significa, por ejemplo, que una ruptura familiar puede desencadenar un proceso excluyente. Pero, para que este avance, tienen que sumarse otros factores, como un padre o una madre con trabajos de baja cualificación y bajo salario, una madre sin empleo o sin experiencia laboral y la existencia de hijos. Una combinación de factores tales desencadena con frecuencia un proceso excluyente. En este hipotético caso, una ruptura familiar es una parte insuficiente (por sí misma no origina siempre exclusión) pero necesaria (sin ella, los otros factores podrían no acarrear exclusión, aunque sean factores de vulnerabilidad) de una combinación de factores que es condición suficiente pero no necesaria de exclusión, pues otras combinaciones de factores puede conducir a situaciones similares de exclusión social.

Esta interesante reflexión metodológica planteada por Zajczyk creo que puede ser abordada mejor con un planteamiento metodológico neofuncionalista, cuyo punto de partida sea, nuevamente, una inversión de términos: en este caso, de la relación entre causa y función, de forma que deje de concebirse el análisis funcional como un caso especial del análisis causal, para pasar a entender este último como un uso del orden funcional (Luhmann 1974a y 1974b).

Esta inversión de la relación entre causalidad y funcionalidad obedece a algo evidente para cualquier investigador social: toda determinación causal lleva consigo una referencia al infinito en diferentes direcciones, porque todo efecto tiene múltiples causas y toda causa tiene múltiples efectos. Además, toda causa puede ser combinada con -o ser sustituida por- otras, de forma que resultan múltiples diferencias en sus efectos. De igual forma, los procesos causales pueden ser descompuestos en un infinito número de partes, por lo que su análisis puede ser continuado hasta el infinito.

Así pues, la búsqueda de una relación constante entre causa y efecto, característica de la concepción clásica de la causalidad, no es aconsejable. Tanto por razones prácticas como teóricas es mejor limitar la invarianza a una causa o a un efecto y fijar una u otro como punto de partida de la búsqueda de relaciones causales equivalentes. $Y$ esto significa que el objetivo de la investigación no debería consistir en la determinación con certeza plena de la relación entre específicas causas y específicos 
efectos, sino en buscar relaciones alternativas. Es decir, se trataría de establecer un "problema" como punto de referencia (en el lado de los efectos o en el de las causas), para, a partir de él y gracias a las posibilidades de comparación que debe proporcionar la teoría, construir un repertorio limitado de resultados alternativos posibles (en el lado de las causas o en el de los efectos).

Aplicado a la investigación de la exclusión social, este "funcionalismo de las equivalencias" implica, por ejemplo, que podemos utilizar una situación de exclusión particularmente frecuente como referencia y, a partir de ella, realizar una búsqueda de relaciones causales equivalentes. Es decir, una vez que se ha establecido una determinada situación como problema de referencia es posible construir un círculo causal relativo a ella, de manera que puedan identificarse diferentes combinaciones causales capaces de producirla y, por eso, susceptibles de considerarse funcionalmente equivalentes, aunque distinguibles por sus efectos secundarios. Desde esta perspectiva, los efectos problemáticos son simples referencias que permiten explorar y ordenar las relaciones entre diferentes causas. También pueden ser empleadas como punto de referencia ciertas causas -por ejemplo, los llamados "factores de vulnerabilidad"- y convertir la explicación de sus efectos en el problema a investigar, de forma que diferentes efectos pueden ser seleccionados, entre sus más frecuentes resultados, como posibles explicaciones de su significado excluyente.

\section{A MOdO DE CONCLUSIONES}

El objetivo primero y fundamental de este artículo ha sido argumentar lo más sólidamente posible y en una clave teórica funcional-estructural, que la inclusión no se da ni puede darse sin exclusión, pues esta es el inseparable reverso de aquella. A ello se ha añadido la idea de que inclusión y exclusión varían en sus formas de materilizarse en función de las formas de diferenciación que ha ido adoptando la sociedad en el curso de su evolución.

Así, se ha sostenido que en la sociedad moderna, funcionalmente diferenciada, no solo la inclusión, sino también la exclusión y las relaciones entre ellas son transferidas de la sociedad a sus subsistemas. Estos, autónomos y poseedores de dinámicas propias de funcionamiento, carecen de una regulación global por parte de la sociedad, a pesar de que los sistemas políticos modernos sean vistos y se vean a sí mismos como depositarios de esta función.

Con ello, ha cambiado radicalmente la estructura de los problemas de exclusión, que ya no pueden ser representados mediante metáforas espaciales del tipo "falta de lugares o sitios sociales", solo adecuados para las sociedades premodernas (segmentadas o estratificadas), en las que la inclusión resulta de la adscripción directa y exclusiva de los seres humanos a uno de los subsistemas sociales. En la sociedad moderna, inclusión y exclusión, pasan a depender de la distribución de oportunidades en cada subsistema, de las tendencias a la acentuación funcional de las disparidades y a la retroalimentación positiva, así como de la muy problemática dependencia múltiple de los sistemas funcionales. Esta última origina que los efectos excluyentes acaecidos en los márgenes de los sistemas produzcan un efecto de integración negativa de las exclusiones -que contrasta con la falta de integración positiva (regulación y solidaridad) de la sociedad-, ya que la exclusión de hecho de un sistema funcional limita lo que puede conseguirse en otros, al punto de que una parte más o menos grande de la población resulte incluso separada residencialmente, y de este modo se convierte en invisible para el resto.

Pero las consecuencias del enfoque teórico utilizado no se limitan al estricto ámbito de la teoría, sino que se extienden también al metodológico. Si todo el edificio teórico del funcional-estructuralismo se sustenta sobre la inversión de la relación entre estructuras y funciones sociales, su correlato metodológico también se asienta sobre otra inversión lógica, en este caso entre los conceptos de "causa" y "función". A partir de ella, el objetivo de una investigación empírica de la exclusión es producir ecuaciones lógicas entre la formulación de un punto de referencia problemático y el tipo de las diferentes posibilidades equivalentes identificadas, bien en el lado de las causas, bien en el de los efectos. $Y$ esta ecuación representa solo un principio analítico y heurístico, pues qué elementos pertenecen a una clase funcional de causas o de efectos de la exclusión es una cuestión empírica, que no puede ser deducida de la formulación de un problema de referencia. Además, como el problema de referencia no explica la existencia fáctica ni la emergencia de concretos resultados, su valor es justamente el contrario: aludir a diferentes posibilidades que deben ser ordenadas en una relación de comparación y sustitución, como demanda la comúnmente aceptada heterogeneidad del espacio de la exclusión social y la diversidad de procesos que conducen a él.

\section{Agradecimientos}

Este artículo es parte de los resultados del proyecto de investigación "Capitalismo de bienestar en el sur de Europa: un análisis comparado", financiado por la ayuda del Ministerio de Economía y Competitividad MINECO-13-CSO2012-33976 (2013-2015). 


\section{NotAs}

1. 'Los 'intocables' no son una casta especial [...], ni un infraestrato disponible para ser explotado. Más bien son un correlato simbólico para la construcción del orden inclusivo mediante preceptos y rituales purificadores [...], que aseguran que los excluidos están omnipresentes y demuestran la necesidad de seguir los mandamientos de pureza" (Luhmann 1997: 621).

2. El enfoque funcional-estructural no ignora, pues, la "condena normativa" que en la semántica moderna distingue al término de exclusión, en beneficio de "simples diferencias sociales", como postula Herzog (2011: 617); por el contrario, da una clara explicación morfo-

\section{RefEREnCIAS BibliográfiCAS}

Bohn, C. 2001. "Inklusionsindividualität und Exklusionsindividualität". Pp. 159-177 en Sinngeneratoren. Fremd- und Selbstthematisierung in soziologisch-historische Perspektive. Alois Hahn zum 60. Geburtstag, editado por C. Bohn y H. Willems. Konstanz: UVK.

Castel, R. 1991. "De l'indigence à l'exclusion, la désafiliation. Précarieté du travail et vulnerabilité relationelle". Pp. 137-168 en Face à l'exclusion. Le model français, editado por J. Danzelot. París: Esprit.

Castel, R. 1995. Les métamorphoses de la question sociale. Une chronique du salariat. París: Fayard.

Dumont, L. 1970. Homo hierarchicus: ensayo sobre el sistema de castas. Madrid: Aguilar 1970.

Evans-Pritchard, E. E. Los nuer. Barcelona: Anagrama.

Fassin, D. 1996. "Exclusion, underclass, marginalidad. Figures contemporaines de la pauvreté urbaine en France, aux Ėtats-Unis et en Amérique latine". Revue Française de Sociologie 37: 37-75. http://dx.doi. org/10.2307/3321945

Fragonard, B. 1993. Cohesion sociale et prévention de l'exclusion. París: La Documentation Française.

Fuchs, P. y D. Scheneider 1995. "Das Hauptmann-vonKöpenick-Syndrom. Überlegungen zur Zukunft funktionaler Differenzierung". Soziale Systeme 2: 7-21.

Fuchs, P. 2000. "Systemtheorie und Soziale Arbeit". Pp. 157176 en Systemtheorie Sozialer Arbeit, editado por R. Merten. Opladen: Leske + Budrich. http://dx.doi. org/10.1007/978-3-322-80858-5_10

Geremek, B. 1976. Les marginaux parisiens aux XIVe et XVe siècles. París: Flamarion.

Geremek, B. 1998. La piedad y la horca. Historia de la miseria y la caridad en Europa. Madrid: Alianza.

Herzog, B. 2011. "Exclusión discursiva. Hacia un nuevo concepto de exclusión social. Revista Internacional de Sociología 69:607-626. http://dx.doi.org/10.3989/ris.2009.12.21

Kanfler, J. 1965. L'exclusion sociale: Étude de la marginalité dans les sociétés occidentales. París: Bureau de Recherches Sociales.

Laparra, M. et al 2007. "Una propuesta de consenso sobre el concepto de exclusión. Implicaciones metodológicas". Revista Española del Tercer Sector 5: 15-57.

Laparra, M. y B. Pérez. 2008. "La exclusión social en España: un espacio diverso y disperso en intensa transformación". Pp. 173-298 en VI Informe sobre exclusión y desarrollo social en España: 2008, editado por Fundación FOESSA. Madrid: Cáritas.

Lenoir, R. 1974. Les exclus. Un Français sur dix. París: Le Seuil. genética de ella, pero para ello es imprescindible que el concepto sociológico no asuma como propia esa carga o fuerza normativa.

3. La alternativa contemplada por Herzog (2011: 617), para que el funcional-estructuralismo supere la supuesta inaplicabilidad de su concepto "radical" de exclusión: limitarlo a concretos contextos dentro de sistemas (por ejemplo, a determinados mercados o segmentos de mercado del sistema económico), es inaceptable para el funcional-estructuralismo, pues, como Herzog señala, acarrearía una graduación multiplicadora y trivializadora de las formas de exclusión.

Lienhardt, G. 1958. "The western Dinka”. Pp. 97-135 en Tribes without rulers: Studies in african segmentary systems, editado por J. Middleton y D. Tait. Londres: Routledge.

Luhmann, N. 1974a. "Funktion und Kausalität". Pp. 9-30 en Soziologische Aufklärung, vol I. Opladen: Westdeutscher.

Luhmann, N. 1974b. "Funktionale Methode und Systemtheorie". Pp. 31-53 en Soziologische Aufklärung, vol. I. Opladen: Westdeutscher.

Luhmann, N. 1995. "Inklusion und Exklusion". Pp. 237264 en Soziologische Aufklärung, vol. VI. Opladen: Westdeutscher.

Luhmann N. 1997. Die Gesellschaft der Gesellschaft. Frankfurt: Suhrkamp.

Mackie, J. L. 1965. "Causes and Conditions". American Philosophical Quarterly 2: 245-264.

Mauri, L. y G. A. Micheli. 1992. Vita di famiglia. Social survey in Veneto. Milán: Franco Angeli.

Nasse, P. 1991. Exclus et exclusions. Connaître les populations et comprendre les processus. París: La Documentation Française.

Parsons, T. 1971. The system of modern societies. Englewood Cliffs NJ: Prentice Hall.

Parsons, T. 1973. "Commentary on Clark". Pp. 299-308 en Perspectives in political sociology, editado por A. Effrat. Indianápolis: Bobbs-Merrill.

Parsons, T. 1974. La sociedad: perspectivas evolutivas y comparativas. México D. F.: Trillas.

Parsons, T. 1977. "Equality and inequality in modern Societies, or social stratification revisited". Pp. 321-380 en Social Systems and the Evolution of Action Theory. Nueva York: Free Press.

Paugam, S. 1991. La disqualification sociale. Essai sur la nouvelle pauvreté. París: PUF.

Paugam, S. 1993. Précarieté et risque d'exclusion en France. París: La Documentation Française.

Piergiovanni, V. 1993. "The itinerant merchant and the fugitive merchant in the Middle Ages". Pp. 81-96 en Of Strangers and Foreigners (Late Antiquity - Middle Ages), editado por L. Mayali y M. M. Mart. Berkeley: University of California at Berkeley.

Posner, R. A. 1980. "A Theory of Primitive Society, with Special Reference to Law". Journal of Law and Economics 23: 1-53. http://dx.doi.org/10.1086/466951

Pound, J. 1986. Poverty and vagrancy in Tudor England. Londres: Routledge.

Radcliffe-Brown, A. R. 1972. Estructura y función en la sociedad primitiva. Barcelona: Península. 
Rosanvallon, P. 1995. La nouvelle question sociale. Repenser l'Etat-providence. París: Le Seuil.

Silver, H. 2007. "The process of social exclusion: the dynamics of an evolving concept". CPRC Working Paper 95. Dpt. of Sociology, Brown University. Providence RI.

Stichweh, R. 2005. Inklusion und Exklusion. Studien zur Gesellschaftstheorie. Bielefeld: Transcript.

JOSE MARÍA GARCíA BLANCO. Catedrático de Sociología y coordinador del Máster Universitario en Políticas Sociales y Bienestar de la Universidad de Oviedo. Especializado en teoría sociológica, en los últimos años ha participado en diversos proyectos de investigación nacionales e internacionales, y publicado diversos libros, capítulos y artículos en editoriales como el Centro de Investigaciones Sociológicas, y en revistas científicas, como Panorama Social, Papers, Política y Sociedad y REIS. Su última publicación fue el artículo "Burbujas especulativas y crisis financieras" (REIS 150, 2015: 71-88).
Subirats, J. dir. 2004. Pobreza y exclusión social. Un análisis de la realidad española y europea. Barcelona: Fundación La Caixa.

Zajczyk, F. 1995. "Between survey and social services analysis: an inquiry on two lines and three levels". Pp. 80101 en Beyond the threshold. The measurement and analysis of social exclusion, editado por G. Room. Bristol: Policy Press. 